\title{
WASSERMANN'S REACTION IN THE SERUM DIAGNOSIS OF SYPHILIS, WITH RESULTS OF MERCURIAL AND 606 TREATMENT.
}

\author{
By Fleet-Surgeon P. W. BASSETT-SMith, C.B., R.N., \\ D.T.M. \& H. (СамB.), ETC. \\ Royal Naval Hospital, Haslar.
}

IN this report are included a series of a thousand cases examined from Jan. 1910 to Jan. 1911, the tests being made weekly at the R. N. Hospital, Haslar. Most of the cases were under observation in the hospital when their clinical characters could be recognised; in many of the cases several tests were applied, week after week, thus making the results obtained of greater value.

The technique used has been that described on previous occasions, the same care having been exercised in making full controls before the test series were examined. In almost every case Fleming's modification of the method was also utilised because it is much more easily employed, takes less time, and requires fewer reagents, but it is however not quite so reliable, erring on the side of over-sensitiveness, whereas Wassermann's technique occasionally fails in the opposite direction. The result obtained from this total of $\mathbf{1 0 0 0}$ cases is shown below :

\begin{tabular}{|c|c|c|c|c|c|}
\hline & Cases & Positive & Negative & \multicolumn{2}{|c|}{ Percentage } \\
\hline Syphilis (primary) & 143 & 106 & $\mathbf{3 7}$ & 74 & positive \\
\hline ", (secondary) & 371 & 348 & 23 & 93.5 & , \\
\hline (tertiary) & 64 & 57 & 7 & 89 & ", \\
\hline Latent syphilis & 54 & 25 & 29 & 46 & ", \\
\hline Parasyphilis & 13 & 13 & - & 100 & $"$ \\
\hline Chancroids & 219 & $1^{*}$ & 218 & $99 \cdot 5$ & negative \\
\hline Gonorrhoea & 23 & - & 23 & 100 & $"$ \\
\hline Other diseases & 113 & $3+$ & 110 & $97 \cdot 4$ & , \\
\hline
\end{tabular}

* The sore was seen eight weeks after infection, it is not known whether the man developed secondary symptoms later.

$\uparrow$ One case of malignant disease of the liver, in a Maltese, with intensely bile stained serum. Two cases of acute malaria.

Journ. of Hyg. xI 
A great number of the primary syphilitic cases were admitted as chancroids, many had mixed infections. It is of the greatest importance in these cases to be able to make a correct diagnosis so as to start specific treatment as soon as possible.

\section{Special reference to negative reactions found in syphilitic cases.}

Of the 143 cases of Primary Syphilis 74\% gave a positive reaction. Of the 37 cases in which the serum gave no indication of the disease, many were very recent and had rarely received any mercurial treatment, several showed the specific spirochaete when examined by the dark ground illumination, and almost all developed secondary symptoms in the hospital; the negative reactions then, if not before, became positive ones.

\section{Illustrative cases.}

(1) Admitted for chancroids, six sores present, contracted on July 12th, first seen on 25th, on Aug. 12th the reaction was negative, on the 18th it had become positive.

(2) Admitted for a sore and bubo contracted on Oct. 1st, first seen on the 21st, on Nov. 3rd, 10th and 16th the reaction was negative, on the 25th it was positive, when the rash began to appear.

(3) Admitted for chancroid which was contracted on July 14th, and first seen on August 4th, on the 14th the reaction was negative, also on Sept. 1st and 9th, becoming positive on the 16th, when the rash was also seen.

(4) Admitted for a chancroid, which was contracted on July 29th, and first seen on Aug. 4th, on the 18th the reaction was negative, but on Sept. 1st it had become positive.

Only 605 of the cases of secondary syphilis gave a negative reaction, in the remainder there had been a considerable elaboration of the immune body in response to the abundance of the infective organism and its toxin. The 23 negative cases were either very early, or they had received very much treatment and the clinical symptoms were slight.

In others, where the disease was latent, negative reactions were most common.

The intermittency of the reaction was frequently seen in those who had received courses of treatment by intra-muscular injections of mercury. 
For instance, in a case giving a well-marked positive reaction, after 14 injections the positive had been replaced by a negative one, which continued for some weeks, then a positive reaction reappeared to be again rendered negative after a further course of seven injections.

This disappearance and reappearance of the reaction makes it impossible to give a definite opinion from a single examination, as to whether the patient is cured or not, a point that should be fully recognised.

At least three negative reactions at three monthly intervals should be obtained before pronouncing a case cured.

During the year special attention has been given to:

(1) The date after infection when the reaction is obtained.

(2) The effect of much treatment on the reaction. This was determined by the study of a series of cases in which the date of infection could be definitely stated, and in which cases it was possible to take many observations. The following table has been drawn up to show the date of the appearance of a positive reaction:

\begin{tabular}{|c|c|c|c|c|}
\hline Case & $\begin{array}{l}\text { Disease } \\
\text { admitted for }\end{array}$ & $\begin{array}{l}\text { Negative } \\
\text { reaction } \\
\text { (in days) }\end{array}$ & $\begin{array}{l}\text { Positive } \\
\text { reaction } \\
\text { (in days) }\end{array}$ & Treatment \\
\hline 1 & Chaneroid & 31 & 37 & Nil \\
\hline 2 & Chancre of face & 28 & 42 & 1 inj. \\
\hline $\mathbf{3}$ & Sp. i & 30 & 45 & Nil \\
\hline 4 & Chancroid & 42 & 49 & " \\
\hline 5 & ," & 28.35 .42 & 49 & $"$ \\
\hline 6 & , & 13.27 & 55 & , \\
\hline 7 & Sp. i & 49 & 56 & " \\
\hline 8 & Chancroid & 49 & 56 & 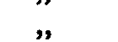 \\
\hline 9 & " & 36.42 .50 & 57 & ," \\
\hline 10 & ", & 27 & 57 & ,, \\
\hline 11 & Sp. i & 36 & 60 & , \\
\hline 12 & Chancroid & 58 & 65 &, \\
\hline
\end{tabular}

This shows that rarely do we obtain a distinctive reaction until some seven or eight weeks after infection, namely not until the disease has become constitutional, when the glands are markedly infected or even when secondary rashes are commencing to appear. This method of diagnosis is of comparatively little help in differentiating a local nonconstitutional sore from a true syphilitic chancre in its early stages.

This is in marked contradistinction to the case in which a positive diagnosis can often be made in early non-treated sores, by demonstrating 
the typical living spirochaete in a few minutes by dark ground illumination, if the proper apparatus is available, or even by means of the Chinese Ink method, which is very simple and frequently as conclusive.

The number of cases having multiple sores of a non-indurated, or only slightly indurated character, that were proved to be specific by the demonstration of the spirochaete at the time (and by their subsequent course) have very much impressed those who have been interested in this work, showing the futility of giving a positive diagnosis by any other means at this early stage.

\section{Effect of Mercurial Treatment.}

From a total of 79 cases that had received over five mercurial injections, 62 still gave a positive reaction.

With over ten injections 35 were still positive; with 20 or more there were still nine positives, and one with 25 , two with 30 , one with 50 and one with 70 injections still remained positive.

Four cases that had been more or less continuously under mercurial treatment for two years, or over, still gave positive reactions.

Negative reactions were obtained in three cases after six injections, in four cases after ten, and 12 injections, in three cases after 16, 18, and 19 injections, in two cases after 20 and 24 injections, in one case after $40,50,64$, and 100 injections respectively.

The amount of mercurial treatment generally required to bring about a constant negative reaction is large; a minimum of three courses of injections being indicated in every case.

Some patients appear to be very refractory to the drug, as shown by their clinical symptoms and by the continued presence of the positive reaction, after $25,30,50$, and 70 injections; in these the use of 606 would appear particularly applicable.

In almost all cases treated by the mouth, without intra-muscular injections, the reaction to the Wassermann test was positive.

Results obtained in cases treated with 606 injections, with the serum reactions given by them, etc.

Two lines of investigation have been followed with regard to the action of this drug on the patients to whom it has so far been administered.

(1) The serum reaction by the complemental deviation method of Wassermann. 
(2) The elimination of the drug by the kidneys.

Eight cases were under observation, all benefited markedly by the remedy; I have noted this personally and so has Staff-Surgeon Shaw, R.N., under whose care the cases have been; to him I am greatly indebted for assistance and co-operation in these examinations.

The blood for the Wassermann reactions was taken together with the usual routine weekly series, the tubes containing the serum from the 606 cases, after numbering, were mixed with the rest of those under examination, and were therefore quite unknown to me until all the readings had been taken.

As 606 is believed to exert an active destructive power upon the specific spirochaete, it is to be expected that a positive Wassermann reaction would soon be replaced by a negative one owing to the diminishing quantity of the immune body called into existence by the infecting organism. It would also appear probable, supposing the dose to be insufficient, that this action would be but little marked, as shown by the continuation of the positive reaction; or if at first apparently effective, yet if the parasitic organisms were not completely destroyed, after a time there would be a renewed growth and further production of the immune body in response, with a return to a positive reaction, indicating that a cure had not been effected, and calling for further treatment.

Following this line of reasoning, the results of the following cases are interesting, but they are too few and incomplete to justify any absolute conclusions.

\section{Cases.}

(1) Late secondary syphilis. Before the administration of 606 the patient had received a good deal of mercury with but little benefit to the symptoms, though his serum gave a negative reaction, this negative reaction continuing after the injection and until the man was discharged.

(2) Severe secondary syphilis. 'The injection was given on Dec. 29th; on Dec. 30th and on Jan. 5th the reaction was strongly positive, on the 12 th it had been converted into a negative, but on the 19th, 26th and Feb. 2nd it became again strongly positive.

(3) Primary and early secondary syphilis. After admission to the hospital the patient's blood gave a positive reaction, on Jan. 3rd he received an injection; on Jan. 5th, 12th, and 19th the reaction was still positive, but on the 26 th it had become converted into a negative one. 
(4) Tertiary syphilis. This patient received an injection on Jan. 8th ; on the 12 th, 19 th, and 26 th positive reactions were obtained.

(5) Primary and early secondary syphilis. This patient's blood was tested on Dec. 30th giving a positive reaction, on Jan. 11th he received an injection of 606, on the 12th, 19th, 26th and Feb. 2nd the reaction was still positive.

(6) Primary and secondary syphilis. This patient received a first injection of 606 on Jan. 12th. When the blood was examined on Jan. 19th and 26 th the reaction was strongly positive; he received a second injection, but on Feb. 2nd the reaction was still positive.

(7) Secondary syphilis. This man's blood was tested on Jan. 5th and $19 \mathrm{th}$, the reaction being positive. On the latter date he received an injection of 606 . There was still a positive reaction after seven days, but in 14 days the reaction became negative.

(8) Primary and early secondary syphilis. On Dec. 17th, when the primary sore alone was present, the reaction obtained was negative. Secondary symptoms developed and on Jan. 23rd he received an injection, on the 26th and Feb. 2nd the reaction was strongly positive.

These observations would indicate that the immune body remains present in the serum after the injection of 606 for at least two weeks, more often for much longer, and that probably one injection is not sufficient to cause a permanent cure of the disease ; further observations are however required.

The excretion of the toxic arsenious drug from the body.

It would seem to be of the greatest importance to know definitely whether the massive dose of arsenic which has been injected is retained or eliminated from the body ; this elimination occurs to a great extent through the kidneys and the metal may be detected in the urine; if the patient is still passing the arsenic out in the urine complete absorption of the drug has not taken place, a further injection of 606 if given then might cause risk of serious toxic symptoms, which danger can be avoided by testing the urine for the metal.

Detection of arsenic in the urine. By the biological test (Gosio's), using Penicillium brevicaulae, a mould which has the property of breaking down organic compounds of arsenic, and giving rise to a distinct garlic-like odour.

This is undoubtedly a very delicate test. 
When the mould is grown upon a media to which a dilute solution of atoxyl or salvarsan has been added the garlic-like odour is very easily detected, but if the media (bread or potato) has added to it urine from a case under treatment, which must be sterilised, infected with the mould and incubated for a day or so, then in my hands the urinary smell produced counteracts or prevents the distinctive odour from being perceived, even in samples of urine which gave a marked quantity of the metal by the chemical test. I have therefore given up this biological method as not being practicable for examination of the urine. Abel and Buttenberg however record that $5 \mathrm{~m}$. of Liq. arsenicalis taken can be detected in the urine.

\section{Chemical analysis with Marsh's test.}

In this test, which is very easily carried out, the well-known ring of metallic arsenic is obtained in the cold part of a capillary tube, through which the heated hydrogen passes.

When the drug is injected it is often absorbed very rapidly, appearing in the urine in large quantities almost immediately after its administration. In one case the urine passed one hour after the injection of 606 gave a very definite deposit; in all cases the first urine passed after the injection contained arsenic, the elimination continued for about 14 days; in two cases examined daily it was detected up to the 13th and 14th day respectively.

It would therefore appear that for the prevention of the toxic effects of the drug on the patient, a period of 14 days should as a rule elapse between the administration of the first and the second injection.

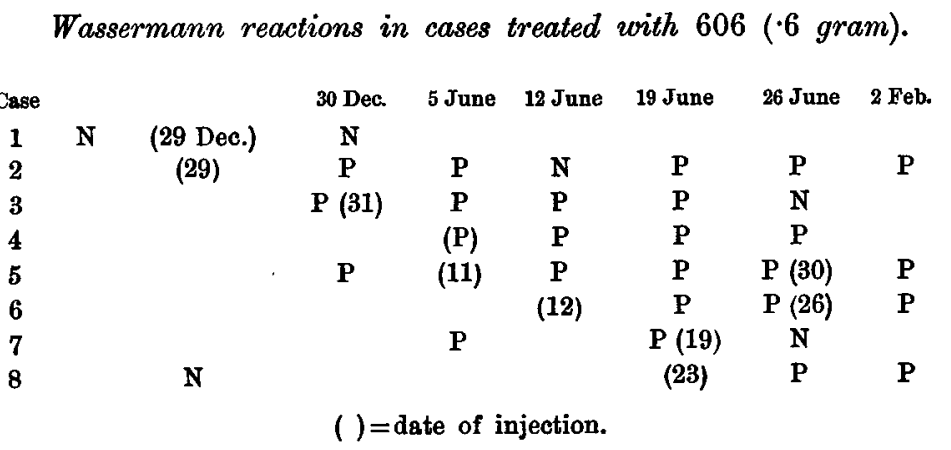


Arsenic in urine in cases treated with 606.

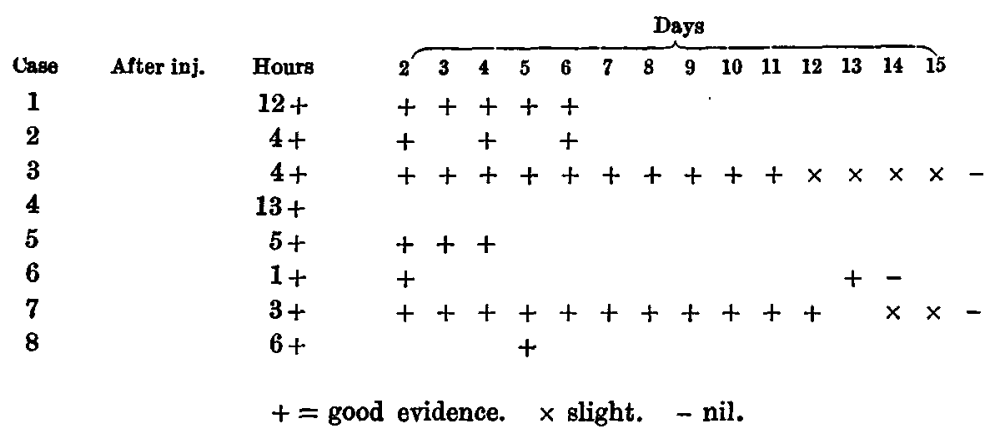

Note. Since this paper was written in January, treatment by salvarsan has been continuously carried out at the Royal Naval Hospital at Haslar, by intravenous instead of intramuscular injections. By the intravenous method the elimination of the arsenic is undoubtedly much more rapid, very great quantities being found in the urine ten minutes after the injection, and rarely after the fourth day. 